\title{
Detection of Point Mutations in DNA BY FLUORESCENCE ENERGY Transfer
}

\author{
Kay M. Parkhurst and Lawrence J. Parkhurst ${ }^{\dagger}$ \\ University of Nebraska, Department of Chemistry, Lincoln, Nebraska 68588-0304 \\ (Paper JBO-057R received Dec. 11, 1995; revised manuscript received Mar. 20, 1996; accepted for publication June 6, 1996)
}

\begin{abstract}
A method has been developed for the rapid and direct identification of a single point mutation in a DNA sequence using fluorescence resonance energy transfer (FRET). The probe was a 16-base oligomer with $5^{\prime}$-bound $\mathrm{x}$-rhodamine and $3^{\prime}$-bound fluorescein $\left(\mathrm{R}^{*} 16^{*} \mathrm{~F}\right)$; the two dyes acted as a donor/acceptor pair for FRET, resulting in a dramatic difference in the fluorescence emission of the $\mathrm{R}^{*} 16^{*} \mathrm{~F}$ in a duplex structure (hybridized to a complementary strand) and as a single strand (melted). This difference was used to obtain the melting temperature $\left(T_{m}\right)$, by spectroscopically following the transition from double to single strand, for the probe hybridized to three different strands: the 16-base complement, the 16-base complement containing a single base mismatch, and the 16-base complementary sequence in the phage DNA M13mp18(+). The $T_{m} \mathrm{~s}$ thus determined for the perfectly base-paired duplexes, with $\mathrm{R}^{*} 16^{*} \mathrm{~F}$ hybridized to the 16-mer complement and to $\mathrm{M} 13$, differed by $2^{\circ} \mathrm{C}$, whereas the $T_{m}$ obtained for $\mathrm{R}^{*} 16^{*} \mathrm{~F}$ hybridized to the mismatched 16-mer complement was $10^{\circ} \mathrm{C}$ lower than that for the perfect duplex. The sharpness of the transition and the ease of detection allow single base mismatches to be reliably detected in nano- and subnanomolar concentrations in less than $1 \mathrm{~h}$ following hybridization. () 1996 Society of Photo-Optical Instrumentation Engineers.
\end{abstract}

Keywords fluorescence energy transfer; mutation detection; hybridization.

\section{INTRODUCTION}

The detection and quantitation of specific sequences of DNA are significant analytical problems of medical importance. Detection involves the use of an oligodeoxyribonucleotide probe that is complementary to the desired sequence and that contains some moiety, such as a radioactive element, that signals the occurrence of hybridization in a filter assay or an electrophoretic gel. Thus, hybridization has been used diagnostically for specific bacterial infections by detection of Mycobacterium tuberculosis genomic DNA, ${ }^{1}$ gonorrhea rRNA, ${ }^{2}$ Chlamydia genomic and plasmid $\mathrm{DNA}^{3}$ and Escherichia coli and Bacillus subtilis rRNA. ${ }^{4}$ Hybridization assays have been developed for viral detection, including cytomegalovirus (CMV) ${ }^{5}$ human papilloma virus (HPV), ${ }^{6}$ and HIV-1. ${ }^{7}$ Rapid progress on the human genome project is ensuring knowledge of many important human DNA sequences.

By combining target amplification with allelespecific oligonucleotides, ${ }^{8}$ very small samples of human DNA can be analyzed for genetic screening and patient counseling. It is important to detect genetic changes associated with the well-known inherited diseases as well as more subtle changes. Weber ${ }^{9}$ has pointed out that cancers at most sites display familial site-specific clustering, ${ }^{10,11}$ and hereditary metabolic variations have been identified that affect the metabolism of known carcinogens. ${ }^{12}$

Address all correspondence to Lawrence J. Parkhurst. E-mail: lparkhurst@unl.edu
The $N$-acetyltransferase (NAT2) gene and gene products, recently under intense study, are a good example. Weber has reviewed the role of polymorphism (from mutations in NAT2) in different rates of in vivo transformations of arylamines and hydrazine carcinogens and drugs. ${ }^{13}$ Point mutations in the NAT2 gene result in lowered levels of the enzyme and divide human populations into so-called "slow" and "rapid" acetylators. Slow acetylators display impaired metabolism not only of useful drugs $^{14}$ but also of arylamine carcinogens present in dyes, pesticides, etc. ${ }^{15}$ While slow acetylators appear to have an increased risk for bladder cancer from arylamine carcinogens, ${ }^{16-18}$ there is evidence for a correlation between rapid acetylation and colorectal cancer. ${ }^{19-21}$ The DNA probes reported here could readily characterize a given sample of DNA as belonging to "slow" or "rapid" acetylator genotypes.

Traditional hybridization methods have employed radioactive probes with separation on filters. ${ }^{22}$ Growing concern over the use of radioactive materials has stimulated a search for other probes that rival the sensitivity of radioactive probes. As early as $1981,{ }^{23}$ biotin was incorporated into an oligodeoxyribonucleotide for use in biotinavidin-linked analyses. Numerous modifications of DNA have been used in the development of similar probes, including links to antibodies, ${ }^{24}$ gold-antibodies, ${ }^{25}$ mercury for double antibody

1083-3668/96/\$6.00 (C) 1996 SPIE 
reactions, ${ }^{26}$ and Eupsoralen ${ }^{27}$ and fluorescent dye links ${ }^{28-30}$ for fluorescence detection of hybridization. These schemes typically allow approximately $10^{5}$ to $10^{6}$ copies of the DNA to be detected. Recent demonstrations $\mathrm{s}^{31-33}$ that single fluorescent molecules can be detected have further increased interest in fluorescence-based schemes for hybridization.

Current methods of mutation detection include denaturing gradient gel electrophoresis (DGGE), single-strand conformational polymorphisms (SSCP), temperature gradient gel electrophoresis (TGGE), the heteroduplex method (HET), ribonuclease cleavage, chemical cleavage of mismatch (CCU), ligase assay, allele-specific amplification (ASA), dideoxy fingerprinting (ddF), and allelespecific oligonucleotides (ASO). ${ }^{34}$ DGGE, SSCP, TGGE, HET, and ddF are frequently used to locate which exons of a gene contain mutations. ASO provides a mechanism for screening for a particular gene mutation. Energy transfer methods based on hybridization such as ASO have other applications as well: at least two companies currently market products for quantifying polymerase chain reactions based on this technology; Biotronics (Aplisensor), ${ }^{35}$ and Perkin-Elmer (Taq Man), ${ }^{36}$ although these technologies differ in details from that discussed here.

We have previously reported ${ }^{37-42}$ the use of Förster resonance energy transfer between donor and acceptor moieties linked to a single DNA strand. In this paper, we extend those studies by demonstrating that a single base substitution can be detected in an oligonucleotide using fluorescence determination of the melting temperature, $T_{m}$, and that $T_{m}$ can be measured in a single circular strand of phage DNA using a short fluorescently labeled oligomer.

\section{METHODS}

\subsection{THEORY}

The probe used in this work and previously ${ }^{37-42}$ was a 16-base deoxyribonucleotide oligomer with $x$-rhodamine covalently bound to the $5^{\prime}$ end and fluorescein to the $3^{\prime}$ end: $x$-rhodamine- $5^{\prime}-$ GTAAAACGACGGCCAG-3'-fluorescein ( $\mathrm{R}^{*} 16^{*} \mathrm{~F}$; see Figure 1). The difference in the fluorescence emission of $R^{*} 16^{*} \mathrm{~F}$ as a single strand or in a duplex structure derives from a difference in an excitonic interaction in the weak-coupling limit, an interaction that varies with the inverse sixth power of the distance between transition moment dipoles of the donor (fluorescein) and acceptor (x-rhodamine) moieties. The theory of such fluorescence (or Förster) resonance energy transfer (FRET) has been treated in numerous papers and texts ${ }^{43-45}$ and only a brief outline will be presented here. The steadystate fluorescence emission intensity of either dye is proportional to its quantum yield, which, neglecting static quenching, is the ratio of the rate constant for fluorescence $\left(k_{F}\right.$, which derives from the Ein-

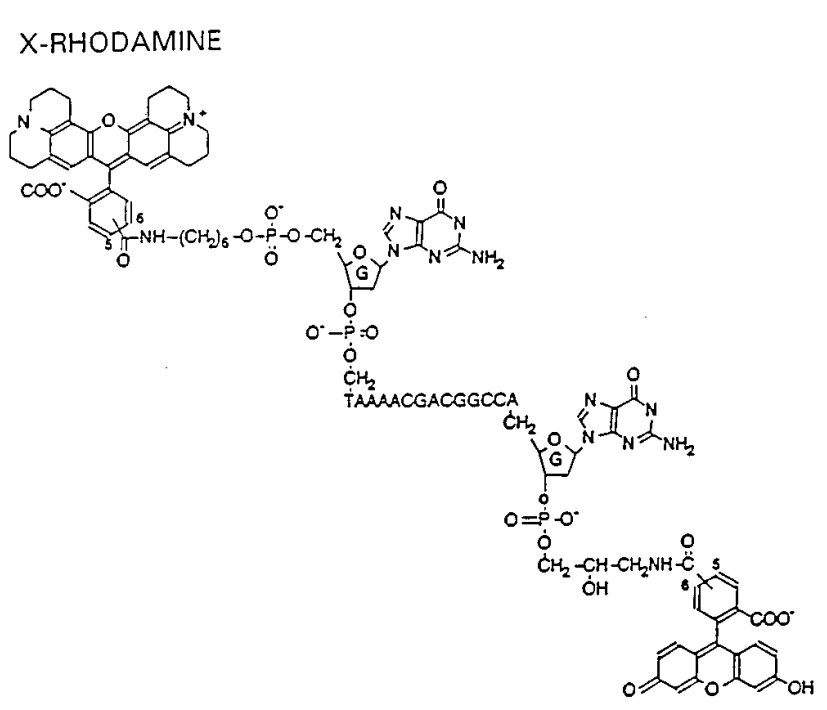

FLUORESCEIN

Fig. I The 16-base oligomer probe. The structures of the 5' $x$-rhodamine and 3 ' fluorescein are shown together with their respective 20- and 16-Å linker arms. In Förster resonance energy transfer, fluorescein acts as the energy donor and x-rhodamine as the acceptor.

stein B coefficient) to the sum of the microscopic rate constants that deactivate the state from which the fluorescence arises (assumed here to be the lowest singlet, $\mathrm{S}_{1}$ ). These processes can be summed as: $k_{F}+k_{I}+k_{t}$, where $k_{\mathrm{I}}$ is the sum of all nonfluorescence processes that deactivate $S_{1}$ in the absence of acceptor and $k_{t}$ is the rate constant for resonance energy transfer. The quantum yield of donor fluorescence in the presence of acceptor is then:

$$
\begin{aligned}
\Phi_{t}=k_{F} /\left(k_{F}+k_{I}+k_{t}\right) & =k_{F} /\left[\left(k_{F}+k_{I}\right)\left(1+X^{6}\right)\right] \\
& =\Phi_{0} /\left(1+X^{6}\right),
\end{aligned}
$$

where $\Phi_{0}$ is the quantum yield in the absence of transfer (absence of acceptor). The quantity $X$ is $R_{0} / R$, where $R$ is the distance between the donor and acceptor, and $R_{0}$ is the donor/acceptor distance at which the rate constant for transfer equals $k_{F}+k_{I} \cdot R_{0}$ can be calculated from the measured spectroscopic properties of the donor and acceptor and applying the usual assumptions with respect to the effective refractive index of the medium separating the donor and acceptor and the rotational averaging of the angular portion of the dipoledipole interaction. It is assumed that $R_{0}$ for fluorescein/x-rhodamine $(60 \AA)$ remains constant throughout the melting process; thus, changes in the steady-state intensity, proportional to $\Phi_{t}$, derive from changes in $R$. (Even if $R_{0}$ should change, it in no way affects our detection of DNA mismatches because the melting process as detected by FRET is essentially one that involves two statesthe hybridized oligo and the free oligo.) 
Hybridization of the $\mathrm{R}^{*} 16^{*} \mathrm{~F}$ to a complementary strand results in a B DNA helical structure with the $3^{\prime}$-fluorescein and the $5^{\prime}$-x-rhodamine positioned at opposite ends. Because the FRET donor and acceptor are relatively far apart, FRET is minimized $\left(k_{t}\right.$ is low); consequently, fluorescein emission is high and rhodamine emission is low (Figure 2, solid line). Upon melting to two single strands, the duplex structure is lost and the more flexible single strand brings the $3^{\prime}$-donor and $5^{\prime}$-acceptor closer together; consequently, FRET is significantly increased, and fluorescein emission decreases and x-rhodamine emission increases (Figure 2, broken line). The melting temperature, $T_{m}$, is the temperature at which the fraction $(f)$ of the total $\mathrm{R}^{*} 16^{*} \mathrm{~F}$ bound to complement is 0.5 . In these experiments, complementary target DNA (C) was in excess over probe $(\mathrm{P})$. The melting process is a sufficiently sharp transition that it may be treated as a two-state process. Let $B$ be the equilibrium concentration of $B$ DNA, and $C_{T}$ and $P_{T}$ be, respectively, total target and probe concentrations. Then the equilibrium constant for helix formation is $K=B /\left[\left(C_{T}-B\right)\left(C_{P}\right.\right.$ $-B)]$ and $f=B / P_{T}$, with all concentrations referenced to a standard state of $1 \mathrm{M}$. The equilibrium constant for $C_{T}>P_{T}$ can then be written as:

$$
\begin{aligned}
K= & {[f /(1-f)]\left[P_{T}(1-f)+\left(C_{T}-P_{T}\right)\right]^{-1}, } \\
& \text { with }[f /(1-f)]=1 \text { at } T_{m} .
\end{aligned}
$$

At $T_{m}, \Delta G=0$, and

$$
\Delta G^{\circ}=-\left(\mathbf{R} T_{m}\right) \ln (K),
$$

where $\mathbf{R}$ is the molar gas constant. If values of $\Delta H^{\circ}$ and $\Delta S^{\circ}$ are available for the given solvent conditions and assumed to be independent of temperature up to $T_{m}$, then, since $\Delta G^{\circ}=\Delta H^{\circ}$ $-T_{m} \Delta S^{\circ}, T_{m}$ can be calculated from:

$$
T_{m}=\Delta H^{\circ} /\left[\Delta S^{\circ}-\mathbf{R} \ln (K)\right],
$$

and is dependent upon probe and target concentrations through $K$, as shown in Eq. (2). The steepness of the transition at the midpoint, $\partial f / \partial T$, gives $\Delta H^{\circ}$ at $T_{m}$ for a true two-state process, and it can be shown that

$$
\Delta H^{\circ}=\mathbf{R} T_{m}^{2}(\partial f / \partial T)\left[4+1 /\left(C_{T} / P_{T}-1 / 2\right)\right] .
$$

Alternatively, as discussed below, $T_{m}$ can be predicted from empirical equations.

\subsection{PROBE}

The DNA probe was designed to be complementary to bases 6291 to 6306 of the 7249-base phage DNA M13mp18(+), one base removed from the restriction site. The choice of a 16-mer was made with several considerations in mind. First, the length was sufficient for the melting temperature to be well above room temperature, ensuring complete

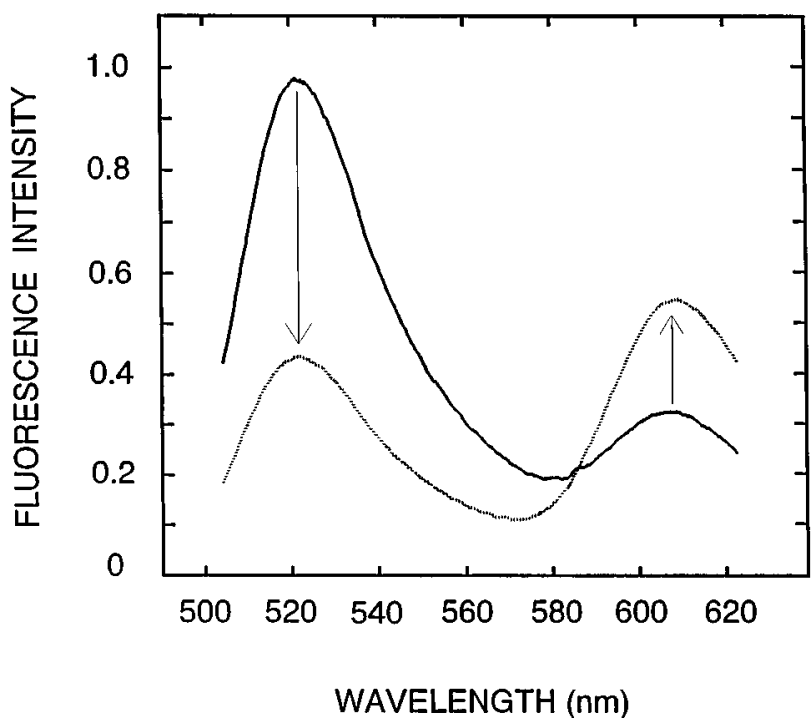

Fig. 2 Steady-state fluorescence emission scans of $5 \mathrm{nM} R * 16 * \mathrm{~F}$ at $20^{\circ} \mathrm{C}$ in $0.18 \mathrm{M} \mathrm{NaCl}$, hybridized to its complement in a duplex structure (solid line) and as a single strand (broken line). Upon melting, the fluorescein emission (520 nm peak) decreases and the $x$-rhodamine emission (610 nm peak) increases, as shown by the arrows. These changes derive from an increased rate of energy transfer between the dyes for the $R^{*} 16 * \mathrm{~F}$ as a single strand (melted) relative to that for the $\mathrm{R}^{*} 16^{*} \mathrm{~F}$ in a duplex structure.

hybridization at $25^{\circ} \mathrm{C}$. Second, for Förster transfer to reflect small changes in interdye distances, the average distance apart should be near $R_{0}$, and modeling studies indicated that with our flexible dye tethers (see Figure 1), the average distance in the duplex would be only $10 \%$ greater than $R_{0}$. Finally, the placement of dyes on bases 1 and 16 resulted in their being located 180 deg apart when viewed down the helix axis, enhancing their separation and the signal change associated with melting.

\subsection{MEASUREMENTS}

The steady-state fluorimeter with computerized data acquisition (Photon Technology International, Inc., Model A-1010) was modified to use as excitation light the 488-nm line of a Coherent (Santa Clara, California) Innova 70-4 $\mathrm{Ar}^{+}$laser. A controllable shutter between the excitation beam and the cuvette ensured that the solution was exposed to excitation light only during the course of a scan, to minimize the photobleaching of fluorescein. The double-labeled oligomer, $\mathrm{R}^{*} 16^{*} \mathrm{~F}$, was from Research Genetics (Huntsville, Alabama), the perfect (CTGGCCGTCGTTTTAC) and single mismatch (CTGGCCGTTGTTTTAC) complements were synthesized in our departmental facility, and the M13mp18(+) was from Sigma (St. Louis, Missouri). The structure of the double-labeled probe, including the $5^{\prime}$ and $3^{\prime}$ dyes and linker arms, is shown in Figure 1. The buffer was $0.01 \mathrm{M} \mathrm{NaPi}, \mathrm{pH} 8,1 \mathrm{~m}$ $M$ EDTA and $0.18 \mathrm{M} \mathrm{NaCl}$, with a total volume of 
$250 \mu \mathrm{l}$ in a stoppered microcuvette. Stock solutions of $\mathrm{R}^{*} 16^{*} \mathrm{~F}$ and complement were diluted to 10 and $20 \mathrm{nM}$, respectively, and allowed to hybridize for $45 \mathrm{~min}$, essentially to completion. (This hybridization time is known from previous work to be adequate. ${ }^{41}$ ) The duplex with the mismatch was monitored spectroscopically for the last $15 \mathrm{~min}$ to ensure that no further change was occurring.

For the $\mathrm{R}^{*} 16^{*} \mathrm{~F} / \mathrm{M} 13$ duplex, stock solutions were diluted to 6.6 and $13.2 \mathrm{nM}$, respectively, and the solution left for $2.5 \mathrm{~min}$ in a heat block (Thermodyne, model 17600 ) at $65^{\circ} \mathrm{C}$. Hybridization occurred as the solution returned slowly to ambient temperature $\left(20^{\circ} \mathrm{C}\right)$ over $1 \mathrm{~h}$. This procedure was used previously and shown not to affect the fluorescent probe. ${ }^{41}$ The cuvette was capped prior to data collection to prevent evaporation at high temperatures. Scans were collected from 502 to $625 \mathrm{~nm}$ every 3 to $4^{\circ} \mathrm{C}$, from 16 to $74.5^{\circ} \mathrm{C}, 16$ to $67^{\circ} \mathrm{C}$, and 20 to $76^{\circ} \mathrm{C}$ for the $\mathrm{R}^{*} 16^{*} \mathrm{~F}$ duplex, mismatch duplex, and M13 duplex, respectively; the solutions were equilibrated for $10 \mathrm{~min}$ at each temperature prior to scanning. (Preliminary work showed no further change after $10 \mathrm{~min}$, which is in good agreement with the procedure reported by Morrison et al. ${ }^{30}$ for melting two singly labeled strands.) The temperature of the solution was controlled using flow through the cuvette holder from a Lauda K-2/R constant-temperature circulating bath, and precise temperatures were determined as follows: for each scan, the temperature of the circulating water was noted. After the experiment, a thermistor was placed in the buffer-filled cuvette to obtain the actual solution temperature for each temperature noted previously. Baseline scans, which included the 583-nm Raman scattering from water and background introduced with the M13, were collected prior to each data set and subtracted from all subsequent scans prior to analysis.

\subsection{DATA ANALYSIS}

The emission scan of the $\mathrm{R}^{*} 16^{*} \mathrm{~F}$ contained two peaks: The peak at $520 \mathrm{~nm}$ was derived from direct absorption by fluorescein of the 488-nm excitation light, modified by FRET. The peak centered at 610 $\mathrm{nm}$ was due primarily to FRET from the fluorescein to the x-rhodamine, with a very small contribution from direct absorbance by x-rhodamine of the excitation light. In this circumstance, the fraction, $f$, of $\mathrm{R}^{*} 16^{*} \mathrm{~F}$ hybridized to complement is ${ }^{41}$ :

$$
f=\sigma(Y) /[1-Y(1-\sigma)],
$$

where

$$
\sigma=I_{\text {rhod }}^{U} / I_{\text {rhod }}^{H}
$$

and

$$
Y=\left(\langle\rho\rangle-\rho^{U}\right) /\left(\rho^{H}-\rho^{U}\right),
$$

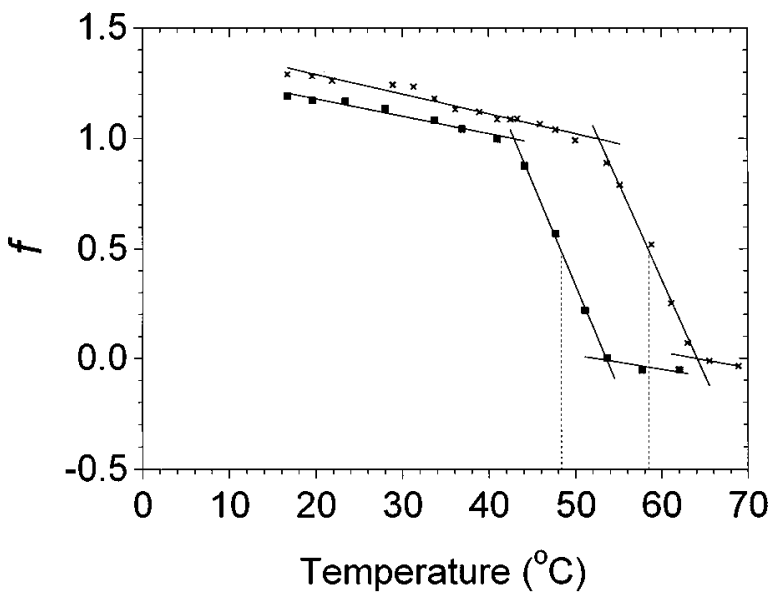

Fig. 3 Melting curves for the $R^{*} 16 * \mathrm{~F}$ duplex, with a perfect complement $(X)$ and to a complement with a single, central $C$ $\rightarrow$ T base substitution ( $\boldsymbol{\square}$ ). The sets of data points correspond to the pre- and postmelting regions and the transition. The $T_{m} s$ are from the midpoints of the duplex $\rightarrow$ single-strand transitions (vertical dashed lines): $58.3^{\circ} \mathrm{C}$ for the duplex of $\mathrm{R}^{*} 16^{*} \mathrm{~F}$ with the perfect complement, and $48.2^{\circ} \mathrm{C}$ for the duplex with the single base substitution. The curves were plotted so that the range of the fraction of hybridized $R^{*} 16 * \mathrm{~F}$, from 1.0 to 0 , was coincident with the ends of the transition region. End fraying and changes in static quenching are likely contributors to the slopes in the pre- and postmelting regions.

and $I_{\text {rhod }}$ is the peak rhodamine emission intensity, $\rho$ is the ratio of the 520- to $610-\mathrm{nm}$ peaks, $\langle\rho\rangle$ is any observed intermediate value of $\rho$, and the superscripts $U$ and $H$ refer, respectively, to unhybridized and hybridized $\mathrm{R}^{*} 16^{*} \mathrm{~F}$. The fraction of bound $R^{*} 16^{*} \mathrm{~F}$ was calculated using Eq. (6) at each temperature measured, and was used to construct the melting curves. The sets of data points on each plot that corresponded to the pre- and postmelting regions and to the melting process were each fit by linear regression and the $T_{m}$ taken as the mid-point of the transition region.

\section{RESUlTS AND DISCUSSION}

The fluorescence emission scans of $R^{*} 16 * \mathrm{~F}$ differ dramatically for the duplexed and single-strand forms (Figure 2). Melting results in a decrease in the fluorescence emission of the fluorescein and an increase in the rhodamine emission, which is consistent with the decrease in the inter-dye distance. Figure 3 shows melting curves for the duplexes formed by $\mathrm{R}^{*} 16^{*} \mathrm{~F}$ hybridized to the perfect 16 -mer complement and to the 16-mer complement with a single $(C \rightarrow T)$ base substitution; the $T_{m} \mathrm{~s}$ for these duplexes are 58.3 and $48.2^{\circ} \mathrm{C}$, respectively. Figure 4 shows the melting curve for the duplex formed between $R^{*} 16^{*} \mathrm{~F}$ and its target sequence in M13mp18(+), with a $T_{m}$ of $56.4^{\circ} \mathrm{C}$. Clearly, the mismatch is easily detected and gives rise to a $\Delta T_{m}$ of $10^{\circ} \mathrm{C}$. This observed decrease in $T_{m}$ is in very good agreement with prediction: the base substitution is 


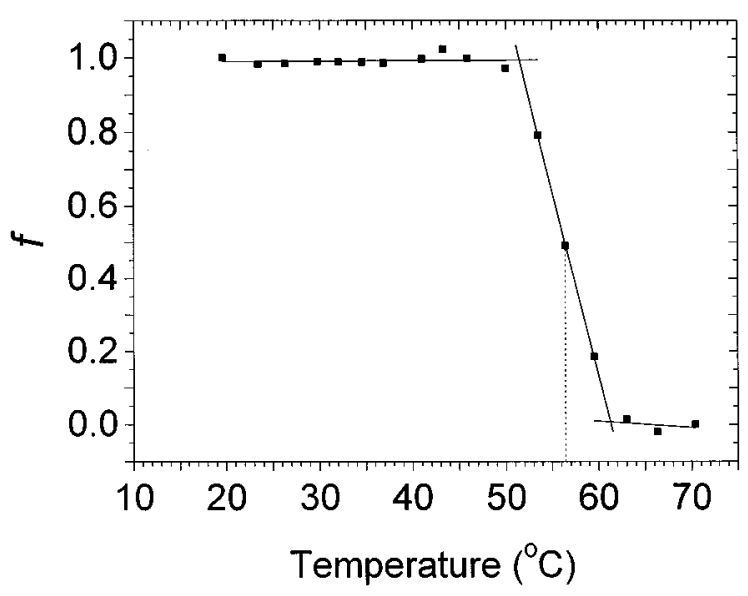

Fig. 4 Melting curve for the $R^{*} 16 * \mathrm{~F}$ probe initially bound to the 7249-base phage DNA, M13mp18(+), which contains the 16base complementary sequence. The $T_{m}$ for the duplex $\rightarrow$ single strand transition, $56.4^{\circ} \mathrm{C}$, is indicated by a vertical dashed line.

expected to affect $T_{m}$ most strongly when it is located near the center of the target sequence ${ }^{46}$ and to decrease the $T_{m}$ by 6 to $9^{\circ} \mathrm{C}\left(\Delta T_{m}=1\right.$ to $1.5^{\circ} \mathrm{C}$ per percent base mismatch in the oligomer probe. ${ }^{46}$ ) The $T_{m}$ of the perfect complement can be predicted rather well from the empirical relationship ${ }^{47,48}$ :

$$
\begin{aligned}
T_{m}\left({ }^{\circ} \mathrm{C}\right)= & 81.5+16.6\left(\log _{10}\left[\mathrm{Na}^{+}\right]\right)+0.41(\% \mathrm{G}+\mathrm{C}) \\
& -600 / N
\end{aligned}
$$

where $N$ is the number of bases in the shorter oligo strand. (In the above equation, the erroneous minus sign in Ref. 48 following 81.5 has been corrected.) For the $\mathrm{R}^{*} 16^{*} \mathrm{~F}$, the predicted $T_{m}$ of $55.5^{\circ} \mathrm{C}$ is in good agreement with the observed values of 56.4 and $58.3^{\circ} \mathrm{C}$ for M13 and the 16-mer complement, respectively. Equation (9), unlike Eq. (4), does not allow a variation of $T_{m}$ with concentrations of probe and target, and thus would not be expected to be accurate within more than a few degrees.

$T_{m}$ is expected to be insensitive to secondary or higher structures around the probe site which have been shown by kinetic studies to characterize M13mp18(+). ${ }^{41}$ A comparison of Figs. 3 and 4 shows that $T_{m}$ is in fact nearly insensitive to the length of the target DNA, particularly considering that the target and probe concentrations were lower for the M13. Assuming that the standard state enthalpy and entropy changes are the same for the two duplexes, Eq. (4) predicts a lower $T_{m}$ for the M13 by about $0.9 \mathrm{deg}$. At this stage of our studies, we cannot say whether the remaining 1-degree difference is insignificant or reflects a perturbation of the target structure. More involved calculations of the melting temperature can be made in which nearest-neighbor interactions are included in $\Delta H^{\circ}$ and $\Delta S^{\circ},{ }^{49}$ using Eq. (4); however, these quantities are not available for our buffer conditions, and the above empirical relationship provides a sufficiently close estimate of $T_{m}$ for the purpose of experimental design. The following thermodynamic quantities for the probe hybridized to the 16-mer complement were calculated from Eqs. (2), (3), and (5) and the fluorescence data: $\Delta G^{\circ}=-49.6 \mathrm{~kJ} / \mathrm{mole}, \Delta H^{\circ}$ $=-373 \pm 21 \mathrm{~kJ} /$ mole of duplex $(-23.3 \mathrm{~kJ} /$ mole of base pair) and $\Delta S^{\circ}=-977 \pm 17 \mathrm{~J} /$ mole-K at $T_{m}$ $=331.5 \mathrm{~K}$. Using values from Breslauer et al. ${ }^{50}$ for the disruption of a CG base pair adjacent to a CG base pair $\left(\Delta H^{\circ}\right.$ and $\Delta S^{\circ}$ more positive by about 50 $\mathrm{kJ} /$ mole and $116 \mathrm{~J} /$ mole-K), a $T_{m}$ of $320.1 \mathrm{~K}$ for the mismatched duplex can be calculated from Eq. (4); this is a decrease in $T_{m}$ of $11.4^{\circ} \mathrm{C}$, which is in remarkable agreement with the $10.1^{\circ} \mathrm{C}$ decrease observed. In this calculation, the changes in $\Delta H^{\circ}$ and $\Delta S^{\circ}$ for lowering $\left[\mathrm{Na}^{+}\right]$from $1 M$ to $0.2 M$ have been neglected.

Several considerations are involved in the design of an optimum probe. The dyes should have limited photobleaching and high quantum yield. Because fluorescein is easily bleached in oxygencontaining solutions, it was necessary to limit exposure of the sample to the exciting laser beam. The dye emission should have limited sensitivity to factors other than changes in distance. Fluorescein emission decreases with $\mathrm{pH}$ (the $\mathrm{pK}$ for the transition from the mono-anion to the highly fluorescent di-anion is $6.7^{50}$ ); consequently, $\mathrm{pH} 8$ sodium phosphate buffer was used, rather than Tris, since the $\mathrm{pH}$ of phosphate buffers is nearly independent of temperature. In order to design an appropriate probe, $R_{0}$ must be calculated or at least estimated. For the studies reported here and previously, ${ }^{40,42}$ we have shown, from the known structure of B-DNA, that the average value of the orientation factor $\left(x^{2}\right)$ must be close to $2 / 3$, which, owing to the lengthy flexible tethers used for dye attachment, is reasonable.

It is convenient to have measurable fluorescence for both the double-labeled oligo as a single strand and in the duplex, as well as sensitivity of the fluorescence to changes in the donor/acceptor distance. Let $\Theta$ be the ratio of the quantum yield in the presence of the acceptor to that in the absence; then $\Theta$ $=\left(1+X^{6}\right)^{-1}=1-\eta$, where $\eta$ is the efficiency of transfer. (The following simple treatment neglects a distribution of distances between donor and acceptor. $^{42}$ ) It is straightforward to show that the maximum sensitivity of $\Theta$ (where sensitivity is defined as $-\mathrm{d} \Theta / \mathrm{d} X)$ to a change in $R$ occurs at $R$ $=R^{*}=(0.946) R_{0}$, for which $-\mathrm{d} \Theta / \mathrm{dX}=1.54$. For $R=R_{0},-\mathrm{d} \Theta / \mathrm{d} X=1.5$, and for either $R^{*}$ or $R_{0}$, a $1 \%$ change in fluorescence intensity corresponds to a change of $0.6 \AA$ in $R$ for $R_{0}=60 \AA$. The sensitivity decreases to 1 on either side of $R_{0}$ (at $R=1.344 R_{0}$ and at $R=0.847 R_{0}$ ) where the respective values of $\Theta$ are 0.855 and 0.269 , a normalized change from 1 to 0.32 . For the 16-mer oligo, distance changes on melting correspond to $R=1.142 R_{0}$ decreasing to $R=0.858 R_{0}$, and the change in normalized fluores- 
cence intensity for the donor (Figure 2) is from 1 to 0.44 . These distance considerations are linked to the desired $\Delta T_{m}$, which increases as the length of the probe decreases [see discussion preceding Eq. (9)] and is maximized for location of the mismatch at or near the center of the probe. The probe length, however, must be sufficient for essentially $100 \%$ hybridization so a full melting transition can be detected even for the mismatch. All of these factors were considered in the present study.

It has been shown ${ }^{51}$ that both optical and calorimetric measurements of the melting transition agree for a 10-mer duplex (having GC base pairs on the ends), showing that the process can be treated to high precision as a two-state process. Intermediates have been detected for single strand melting, however, ${ }^{52}$ and there may be instances where bases, particularly at the ends of the duplex, are unpaired before full strand separation. On the other hand, the large change in fluorescence emission reported here would be expected only after actual strand separation and thus might be expected to lead to the appearance of a sharper transition than that observed by absorbance. Monitoring absorbance changes associated with melting is not feasible for duplexes between short oligomers and long strands of DNA due to the overwhelming background from the long strand.

The long DNA strand studied here was singlestranded. Special hybridization techniques are required with DNA that is originally duplex, ${ }^{41}$ yet the melting process, once the probe has bound, should closely resemble that reported here; it is essentially independent of higher order structure in the vicinity of the target, owing to the large $\Delta H^{\circ}$ of melting. In practice, one would titrate the probe (in order to maintain nearly constant fluorescence intensity) with DNA to determine the number of copies of the target, and then carry out the melting experiments as described above. Once hybridization is achieved, having an estimate of $T_{m}$ from Eq. (9), one could start the temperature ramp about 10 deg below the expected $T_{m}$ for the mismatch and complete the analysis within $1 \mathrm{~h}$. The sharp transition detected by FRET using the double-labeled oligonucleotide allows a rapid and clear determination of whether a mismatch is present at the predicted location in the target. The technique should allow rapid detection of point mutations for NAT2 and other genes in human DNA.

\section{Acknowledgment}

This work was supported by National Institutes for Health grant DK36288 to the Center for Biotechnology, University of Nebraska-Lincoln.

\section{REFERENCES}

1. K. D. Eisenach, J. T. Crawford, and J. H. Bates, "Repetitive DNA sequences as probes for Mycobacterium tuberculosis," $\mathrm{J}$. Clin. Microbiol. 26, 2240-2245 (1988).

2. M. E. Harper, C. Gonzalez, M. S. You, C. V. Gegg, D.
Kranig-Brown, Y. Y. Yang, R. A. Respess, and P. R. Roeder, "A rapid non-isotopic DNA probe test for the direct detection of Neisseria gonorrhoeae in clinical specimens," Abstr. Am. Soc. Microbiol. Ann. Meeting, p. 338 (1988).

3. A. Palva, K. Korpela, A. Lassus, and M. Ranki, "Detection of Chlamydia trachomatis from genito-urinary specimens by improved nucleic acid sandwich hybridization,", FEMS Microbiol. Lett. 40, 211-217 (1987).

4. C. A. Miller, W. L. Patterson, P. K. Johnson, C. T. Swartzell, F. Wogoman, J. P. Albarella, and R. J. Carrico, "Detection of bacteria by hybridization of rRNA with DNA-latex and immunodetection of hybrids," J. Clin. Microbiol. 26, 1271-1276 (1988).

5. A. A. Scott, K. A. Walker, L. M. Hennigar, C. H. Williams, J. P. Manos, and T. Gansler, "Detection of cytomegalovirus in shell vial cultures by using a DNA probe and early nuclear antigen monoclonal antibody," J. Clin. Microbiol. 26, 18951897 (1988).

6. S. P. Goltz, J. A. Todd, and H. L. Yang, "A rapid DNA probe test for detecting HPV in biopsy specimens," Am. Clin. Products Rev., Dec. 16-19 (1987).

7. J. Richards, M. Collins, P. Groody, C. Pritchard, J. Thompson, and D. Gillespie, "Development of a sensitive hybridization assay for detection of human immunodeficiency virus RNA," Abstr. Am. Soc. Microbiol. Ann. Meeting, p. 331 (1988).

8. R. Higuchi, C. H. von Beroldingen, G. F. Sensabaugh, and H. A. Erlich, "DNA typing from single hairs," Nature 332, 543-546 (1988).

9. W. Weber, in Hereditary Cancer and Preventive Surgery, W. Weber, U. T. Laffer, and M. Dürig, Eds., pp. 8-11, Karger, Basel (1990).

10. H. T. Lynch and T. Hirayama, Eds., Genetic Epidemiology of Cancer, CRC Press, Boca Raton, FL (1989).

11. H. J. Müller and W. Weber, Familial Cancer, Karger, Basel (1985).

12. N. Caporaso, L. W. Picle, S. Bale, R. Ayesh, M. Hetzel, and J. Idle, "The distribution of debrisoquine metabolic phenotypes and implications for the suggested association with lung cancer risk," Genet. Epidemiol. 6, 517-524 (1989).

13. W. Weber, The Acetylator Genes and Drug Response, Oxford Univ. Press, New York (1987).

14. U. A. Meyer "Genetic polymorphisms of drug metabolism," Fundamen. Clin. Pharmacol. 4, 595-615 (1990).

15. National Academy of Sciences, Aromatic Amines: an Assessment of the Biological and Environmental Effects, National Academy Press, Washington, DC (1981).

16. D. W. Hein, "Acetylator genotype and arylamine-induced carcinogenesis," Biochem. Biophys. Acta 949, 37-66 (1988)

17. D. W. Nebert, "Role of genetics and drug metabolism in human cancer risk," Mutation Res. 247, 267-281 (1991).

18. I. Roots, N. Drakoulis, and J. Brockmoller, "Polymorphic enzymes and cancer risk: concepts, methodology, and data review," in Pharmacogenetics of Drug Metabolism, W. Kalow, Ed., pp. 815-841, Pergamon Press, New York (1992).

19. J. D. Wohlleb, C. F. Hunter, B. Blass, F. F. Kadlubar, D. Z. J. $\mathrm{Chu}$, and N. P. Lang. "Aromatic amine acetyltransferase as a market for colorectal cancer: environmental and demographic associations," Int. J. Cancer 46, 22-30 (1990).

20. N. P. Lang, Z. J. Chu, C. F. Hunter, D. C. Kendall, T. J. Flammang, and F. F. Kadlubar, "Role of aromatic amine acetyltransferase in human colorectal cancer," Arc. Surg. 121, 1259-1261 (1986).

21. K. F. Ilett, B. M. David, P. Detchon, W. M. Astleden, and R. Kwa, "Acetylation phenotype of colorectal carcinoma," Cancer Res. 47, 1466-1469 (1987).

22. B. D. Hames and S. J. Higgins, Eds., Nucleic Acid Hybridization, IRL Press, Washington, DC (1985).

23. P. R. Langer, A. A. Waldrop, and D. C. Ward, "Enzymatic synthesis of biotin-labeled polynucleotides: novel nucleic acid affinity probes," Proc. Natl. Acad. Sci. U.S.A. 78, 66336637 (1981).

24. P. Tchen, R. P. P. Fuchs, E. Sage, and M. Leng, "Chemically modified nucleic acids as immuno-detectable probes in hybridization experiments," Proc. Natl. Acad. Sci. U.S.A. 81, 3466-3470 (1984). 
25. S. Tomlinson, A. Lyga, E. Huguenel, and N. Dattagupta, "Detection of biotinylated nucleic acid hybrids by antibodycoated gold colloid," Anal. Biochem. 171, 217-222 (1988).

26. A. H. N. Hopman, J. Wiegant, G. I. Tesser, and P. Van Dujin, "A non-radioactive in situ hybridization method based on mercurated nucleic acid probes and sulfhydryl-hapten ligands," Nucleic Acids Res. 14, 6471-6488 (1986).

27. A. Oser, W. K. Roth, and G. Valet, "Sensitive nonradioactive dot-blot hybridization using DNA probes labeled with chelate group substituted psoralen and quantitative detection by europium ion fluorescence," Nucleic Acids Res. 16, 1181-1196 (1988).

28. L. M. Smith, S. Fung, M. W. Hunkapiller, T. J. Hunkapiller, and L. E. Hood, "The synthesis of oligonucleotides containing an aliphatic amino group at the 5' terminus: synthesis of fluorescent DNA primers for use in DNA sequence analysis," Nucleic Acids Res. 13, 2399-2412 (1985).

29. J. Wiegant, T. Ried, P. M. Nederlof, M. van der Ploeg, H. J. Tanke, and A. K. Raap, "In situ hybridization with fluoresceinated DNA," Nucleic Acids Res. 19, 3237-3241 (1991).

30. L. E. Morrison, T. C. Halder, and L. M. Stols, "Solutionphase DNA assay using fluorescent probes," Anal. Biochem. 183, 231-244 (1989).

31. M. Ishikawa, K. Hirano, T. Hayakawa, S. Hosoi, and S. Brenner, "Single-molecule detection by laser-induced fluorescence technique," Jpn. J. Appl. Phys. 33, 1571-1576 (1994).

32. M. Eigen and R. Rigler, "Sorting single molecules: application to diagnostics and evolutionary biotechnology," Proc. Natl. Acad. Sci. U.S.A. 91, 5740-5747 (1994).

33. S. Nie, D. T. Chiu, and R. N. Zare, "Probing individual molecules with confocal fluorescence microscopy," Science 266, 1018-1021 (1994)

34. I. Dianzani, C. Camaschella, and A. Ponzone, "Dilemmas and progress in mutation detection," Trends Genet. 9, 403405 (1993).

35. Amplisensor Assay, Biotronics, Lowell, MA.

36. TaqMan PCR Detection System, Perkin-Elmer, Foster City, CA.

37. K. M. Parkhurst and L. J. Parkhurst, "Fluorescence studies of oligonucleotide-DNA hybridization in solution and of oligonucleotide end to end distance distributions," Abstr., 11th Intern. Congr. Photobiology, p. 258, Japan Photochem. Soc., Kyoto, Japan (1992).

38. K. M. Parkhurst and L. J. Parkhurst, "Kinetic studies of oligonucleotide-DNA hybridization in solution by fluores- cence energy transfer," Abstr. Biophys. J. 64, A266 (1993).

39. K. M. Parkhurst and L. J. Parkhurst, "FRET Studies of OigoDNA Interactions," Abstr. Biophys. J. 66, A154 (1994).

40. L. J. Parkhurst and K. M. Parkhurst, "Changes in the end-to end distance distribution in an oligonucleotide following hybridization," Proc. SPIE 2137, 475-483 (1994).

41. K. M. Parkhurst and L. J. Parkhurst, "Kinetic Studies by fluorescence resonance energy transfer employing a doublelabeled oligonucleotide: hybridization to the oligonucleotide complement and to single stranded DNA," Biochemistry 34, 285-292 (1995).

42. K. M. Parkhurst and L. J. Parkhurst, "Donor-acceptor distance distributions in a double-labeled fluorescent oligonucleotide both as a single strand and in duplexes," Biochemistry 34, 293-300 (1995).

43. C. Cantor and P. Schimmel, Biophysical Chemistry. II. Techniques for the Study of Biological Structure and Function, pp. 448-454, W. H. Freeman, San Francisco (1980).

44. J. Lakowicz, Principles of Fluorescence Spectroscopy, Plenum Press, New York (1983).

45. T. Förster, Fluoreszenz Organischer Verbindungen, Vandenhoeck and Ruprecht, Göttingen (1951).

46. J. Sambrook, E. F. Fritsch, and T. Maniatis, Molecular Cloning, a Laboratory Manual, 2nd ed., Book 2, pp. 11-47, Cold Spring Harbor Laboratory Press, Cold Spring Harbor, NY (1989).

47. B. D. Hames and S. J. Higgins, Nucleic Acid Hybridization, p. 80, IRL Press, Washington, DC (1985).

48. J. Sambrook, E. F. Fritsch, and T. Maniatis Molecular Cloning, a Laboratory Manual, 2nd ed., Book 2, pp. 11-46, Cold Spring Harbor Laboratory Press, Cold Spring Harbor, NY (1989).

49. K. J. Breslauer, R. Frank, H. Blocker, and L. Marky, "Predicting DNA duplex stability from the base sequence," Proc. Natl. Acad. Sci. U.S.A. 83, 3746-3750 (1986).

50. L. Lindqvist, "A flash photolysis study of fluorescein," Arkiv f. Kemi 16, 79-138 (1960).

51. Y.-W. Park and K. J. Breslauer, "A spectroscopic and calorimetric study of the melting behaviors of a "bent"

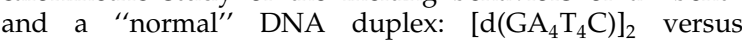
$\left[\mathrm{d}\left(\mathrm{GT}_{4} \mathrm{~A}_{4} \mathrm{C}\right)\right]_{2}, "$ Proc. Natl. Acad. Sci. U.S.A. 88, 1551-1555 (1991).

52. C. Cantor and P. Schimmel, Biophysical Chemistry III. The Behavior of Biological Macromolecules, pp. 1131-1132, W. H. Freeman, San Francisco. 